\title{
VIRTUALIZAÇÃO E CLOUD COMPUTING NAS PME'S EM PORTUGAL
}

\author{
Miguel Cássio Ferreira Lavrador e António Jorge Gonçalves de Gouveia \\ Universidade de Trás-os-Montes e Alto Douro, Quinta de Prados, 5000-801 Vila Real, Portugal
}

\begin{abstract}
RESUMO
Num mundo altamente desenvolvido a nível tecnológico, os sistemas computacionais desempenham um papel fundamental. Utilizados em diversos contextos, os computadores são utilizados por todas as pessoas, quer para realizarem tarefas banais como para tarefas complexas e exigentes.

O cloud computing e a virtualização são temas bastante investigados, devido a algumas características intrínsecas aos seus conceitos, como a redução de custos, o melhor aproveitamento do espaço físico da empresa e a sua praticidade (Gong, Liu, Zhang, Chen, \& Gong, 2010). Nas organizações, estas novas tecnologias estão no centro do seu desenvolvimento e crescimento (Dias, Rodrigues, \& Pires, 2012). Assim, perceber até que ponto as empresas portuguesas conhecem, utilizam e usufruem de diversas tecnologias como ferramentas de trabalho, foi uma das grandes questões que motivou a realização deste trabalho.
\end{abstract}

\section{PALAVRAS-CHAVE}

Virtualização, Cloud Computing, Serviços de Outsourcing, PME's

\section{INTRODUÇÃO}

Este trabalho serve o propósito de divulgar os resultados obtidos nos inquéritos realizados pelas diversas empresas portuguesas e, além disso, analisar e confrontar esses resultados com a informação acerca da adoção de virtualização e de cloud computing nas PME's com o intuito de verificar o impacto que a sua utilização possui no crescimento deste tipo de empresas.

Futuramente pretende-se ainda comparar os resultados com as realidades e o nível de preparação para a adoção e crescimento de serviços de cloud computing de outros países de todo o mundo.

\section{CONCEITOS}

A virtualização consiste na emulação de ambientes isolados capazes de utilizar diferentes sistemas operacionais (SOs) na mesma máquina, o que proporciona uma camada de abstração dos verdadeiros recursos da máquina (Coelho, Calzavara, \& di Lucia, 2008), e capacita cada sistema com hardware virtual. Existem várias formas de virtualização, tais como: virtualização de servidor, virtualização de aplicação, virtualização de desktop, virtualização de rede, virtualização de apresentação e virtualização de dados.

Por sua ver, o cloud computing é um conjunto de princípios e abordagens que disponibiliza aos utilizadores recursos de rede, armazenamento, plataforma e aplicações através da nuvem (Saleem, 2017). O cloud computing utiliza a conetividade e a dispersão em grande escala da internet pelo mundo para hospedar os mais diversos recursos, permitindo ao utilizador o acesso a eles através de um dispositivo com um navegador e conexão à internet (Giraldo, 2018).

Hoje em dia, o cloud computing oferece uma ampla gama de serviços, ferramentas e recursos para fornecer ao cliente / empresa o serviço que melhor se adapta às suas necessidades específicas. Entre outros, destacam-se três modelos: SaaS - Software como Serviço, PaaS - Plataforma como Serviço e IaaS - Infraestrutura como Serviço. Ao implementar uma infraestrutura de computação em nuvem, é importante considerar o tipo de informação e dados que são armazenados, bem como os processos de negócio 
e o nível de visão desejado pela empresa que irá utilizá-la (Ruschel, Zanotto, \& da Mota, 2010). Sendo assim, os diferentes tipos de implementação mais utilizados são: privada, pública e híbrida.

Estes conceitos estão intimamente ligados e complementam-se, isto é, o cloud computing surge como forma de monetizar a partilha de recursos - virtualização - ao disponibilizá-los, como serviços. Um ambiente virtualizado traz benefícios em vários níveis, leva a melhores resultados de implementação de nuvem e tem um grande impacto na economia da organização (Lavrador \& Gouveia, 2020).

\section{VIRTUALIZAÇÃO E CLOUD COMPUTING NAS PME'S EM PORTUGAL}

Neste ponto iremos apresentar os resultados obtidos nas entrevistas realizadas às diversas empresas portuguesas e, além disso, analisar e confrontar esses resultados com a informação acerca da adoção de virtualização e de cloud computing nas PME's. Uma vez que a investigação nesta área é um pouco escassa, este estudo é um contributo para o preenchimento desta lacuna, através da construção e validação empírica de um modelo que pretende explicar a forma como a virtualização e o cloud computing são vistos pelas empresas portuguesas e a sua influência no crescimento e desenvolvimento das PME's.

Em seguida é descrita a metodologia utilizada neste estudo empírico. Começa-se por definir a população do estudo e por realizar uma caracterização da amostra e, posteriormente, apresenta-se os resultados obtidos.

\subsection{Descrição do Estudo}

A população do estudo, ou seja, o conjunto de elementos sobre os quais se vai debruçar a investigação, engloba empresas de todas as dimensões, com maior incidência nas empresas de pequena e média dimensão, todas elas portuguesas.

O estudo empírico teve por base uma amostra aleatória, uma vez que os inquéritos foram enviados por e-mail para as empresas que tinham o seu contacto online ou que estavam presentes em plataformas/sites de diretórios de empresas. Ao todo, foram enviados 2191 e-mails, dos quais 668 não foram entregues, uma vez que a informação estava desatualizada ou incorreta. Dos 1523 contactos restantes, foram obtidas apenas 540 respostas, divididas pelas várias categorias de dimensão da empresa existentes (Tabela 1), sendo esta a população do estudo.

Tabela 1. Categoria de dimensão da empresa

\begin{tabular}{c|ccccc}
$\begin{array}{c}\text { Dimensão da } \\
\text { empresa }\end{array}$ & Micro & Pequena & Média & Grande & Total \\
\hline $\begin{array}{c}\mathbf{N}^{\mathbf{0}} \text { de } \\
\text { empresas }\end{array}$ & 276 & 108 & 84 & 72 & 540 \\
$(51,1 \%)$ & $(20 \%)$ & $(15,6 \%)$ & $(13,3 \%)$ & $(100 \%)$
\end{tabular}

O instrumento de recolha de dados utilizado foi um inquérito dividido em duas partes principais:

(i) a primeira, que incidiu nas organizações que utilizam tecnologias de virtualização e serviços de cloud computing, tem o intuito de perceber quais os serviços utilizados, há quanto tempo os utilizam, os modelos implementados, a importância atribuída às vantagens e desvantagens e a opinião e satisfação geral acerca da sua utilização;

(ii) a segunda, que contempla as organizações que não utilizam tecnologias de virtualização e serviços de cloud computing, tem como objetivo verificar o nível de conhecimento e divulgação destas por parte das empresas portuguesas.

Os potenciais inquiridos foram contactados por e-mail, sendo solicitados a responder ao questionário a partir de um link para a página onde se encontrava alojado o mesmo. O link de acesso ao questionário esteve disponível durante cerca de 9 meses, entre fevereiro de 2019 e novembro de 2019. 


\subsection{Análise de Resultados}

O cloud computing aliado às técnicas de virtualização traz inúmeros benefícios para as empresas, constituindo uma importante inovação tecnológica. Contudo, a utilização destes serviços é ainda diminuta em Portugal, de acordo com o BSA Global Cloud Computing Scorecard.

Em primeiro lugar, verificámos o nível de utilização e de conhecimento destas tecnologias e serviços e percebemos que ambos são muito reduzidos (Tabela 2), o que demonstra alguma carência na divulgação das mais-valias e das potencialidades da virtualização e do cloud computing. Esta afirmação é confirmada através do estudo sobre a adoção de serviços de cloud computing pelas empresas portuguesas efetuado por Nuno Fortes, José Henrique Pereira e João Fontes da Costa, que investigaram a influência dos esforços de marketing na adoção destes serviços, enquanto potenciadores da intenção de utilização. Os resultados apontam para um impacto significativo dos esforços de marketing nas condições facilitadoras de adoção de serviços de cloud, uma vez que incentivam as perceções de segurança e confiança das empresas (Fortes, Pereira, \& da Costa, 2016), provocando uma maior adesão ao serviço.

Tabela 2. Resultados dos inquéritos

\begin{tabular}{|c|c|c|c|}
\hline $\begin{array}{c}\text { Utiliza na sua } \\
\text { empresa } \\
\text { virtualização ou } \\
\text { cloud computing? }\end{array}$ & $\begin{array}{c}\text { Sim } \\
(\mathbf{3 7 , 8 \%}) \\
\text { Não } \\
(62,2 \%)\end{array}$ & $\begin{array}{c}\text { Conhece outras } \\
\text { empresas que } \\
\text { utilizem? }\end{array}$ & $\begin{array}{c}\text { Sim } \\
(\mathbf{3 5 , 7 \%}) \\
\text { Não } \\
(64,3 \%)\end{array}$ \\
\hline $\begin{array}{l}\text { Está familiarizado } \\
\text { com os conceitos? }\end{array}$ & $\begin{array}{c}\text { Sim } \\
(31,2 \%) \\
\text { Não } \\
(68,8 \%)\end{array}$ & $\begin{array}{c}\text { Conhece as suas } \\
\text { vantagens e } \\
\text { desvantagens? }\end{array}$ & $\begin{array}{c}\text { Sim } \\
(29,2 \%) \\
\text { Não } \\
(70,8 \%)\end{array}$ \\
\hline
\end{tabular}

A virtualização e o cloud computing são relativamente recentes e através da tabela que se segue (Tabela 3) é possível confirmar esta afirmação, uma vez que da sua análise percebe-se que apenas uma reduzida quantidade de empresas as utilizam há mais de 10 anos $(9,3 \%)$.

Tabela 3. Anos de utilização de virtualização e cloud computing

\begin{tabular}{c|ccccc}
$\begin{array}{c}\text { Anos de } \\
\text { utilização }\end{array}$ & $\begin{array}{c}\text { Menos } \\
\text { de 1 }\end{array}$ & $\mathbf{1 - 3}$ & $\mathbf{4}$ - 6 & $\mathbf{7 - 9}$ & $\begin{array}{c}\text { Mais } \\
\text { de 10 }\end{array}$ \\
\hline $\begin{array}{c}\mathbf{N}^{\mathbf{0}} \text { de } \\
\text { empresas }\end{array}$ & $\begin{array}{c}21 \\
(10,3 \%)\end{array}$ & $\begin{array}{c}33 \\
(16,2 \%)\end{array}$ & $\begin{array}{c}108 \\
(52,9 \%)\end{array}$ & $\begin{array}{c}23 \\
(11,3 \%)\end{array}$ & $\begin{array}{c}19 \\
(9,3 \%)\end{array}$
\end{tabular}

No que respeita ao orçamento anual dedicado às infraestruturas de TI/SI pelas PME's, a Tabela 4 mostra um investimento relativamente baixo, $53 \%$ das empresas dedicam menos de $10 \%$ do seu orçamento anual às TI/SI, isto porque as empresas não têm a perceção de todas as potencialidades dos serviços de cloud computing e têm a ideia de que a relação entre o custo e os benefícios não compensa ou não se adequa ao seu negócio. Este raciocínio leva os responsáveis a não enveredar por soluções deste tipo, colocando de parte a oportunidade para a mudança de paradigma e inovação tecnológica. Além disto, o orçamento disponibilizado para serviços de cloud computing nas empresas está também dependente da sua dimensão uma vez que, empresas com maior dimensão desfrutam de um maior encaixe financeiro e, por sua vez, possuem maior liberdade de investimento.

Tabela 4. Orçamento anual dedicado às TI/SI

\begin{tabular}{c|ccccc} 
\% de Orçamento & Menos de 5 & $\mathbf{5 - 1 0}$ & $\mathbf{1 0}-\mathbf{2 5}$ & $\mathbf{2 5}$ - 35 & Mais de 35 \\
\hline \multirow{2}{*}{$\mathbf{N}^{\mathbf{o}}$ de empresas } & 26 & 83 & 76 & 13 & 6 \\
& $(12,7 \%)$ & $(40,7 \%)$ & $(37,3 \%)$ & $(6,4 \%)$ & $(2,9 \%)$
\end{tabular}

Através das tabelas 5 e 6, respetivamente, apresentamos os serviços mais utilizados e o modelo de cloud computing mais implementado pelas empresas portuguesas. É importante referir que uma empresa pode aderir a vários serviços em simultâneo de acordo com as exigências do seu negócio. O modelo de implementação é, também, escolhido de acordo com o tipo de negócio e com a infraestrutura montada. 
Tabela 5. Serviços de cloud computing implementados

\begin{tabular}{c|cccccc} 
Serviços & SaaS & PaaS & IaaS & STaaS & SECaaS & DBaaS \\
\hline $\begin{array}{c}\mathbf{N}^{\mathbf{0}} \mathbf{d e} \\
\text { empresas }\end{array}$ & 121 & 38 & 99 & 105 & 35 & 69 \\
$(59,3 \%)$ & $(18,6 \%)$ & $(48,5 \%)$ & $(51,5 \%)$ & $(17,2 \%)$ & $(33,8 \%)$
\end{tabular}

Tabela 6. Modelos de cloud computing implementados

\begin{tabular}{c|ccc} 
Modelos & Privada & Pública & Híbrida \\
\hline $\mathbf{N}^{\mathbf{0}}$ de & 132 & 12 & 60 \\
empresas & $(64,7 \%)$ & $(5,9 \%)$ & $(29,4 \%)$
\end{tabular}

Também foram objeto de estudo as vantagens e desvantagens associadas à implementação de virtualização e cloud computing, mais precisamente a importância dada a estas pelas PME's. Com isto pretende-se dar a conhecer às empresas que ainda não utilizam este tipo de técnicas e serviços as suas potencialidades de forma a motivar a sua adesão e de certa forma, alargar também a sua disseminação no mundo empresarial.

Sendo assim as tabelas 7 e 8 traduzem essa importância através de cinco níveis, divididos em três categorias principais: pouco importante, importante, e muito importante. Com base nestes dados observamos que a segurança, a capacidade de armazenamento e as atualizações automáticas são as vantagens mais reconhecidas pelas empresas e que as vulnerabilidades de segurança e as perdas de dados ou informações são as suas maiores preocupações.

Tabela 7. Importância das vantagens do cloud computing

\begin{tabular}{|c|c|c|c|}
\hline Vantagens & $\begin{array}{l}\text { Pouco Importante } \\
(1-2)\end{array}$ & $\begin{array}{l}\text { Importante } \\
\text { (3) }\end{array}$ & $\begin{array}{c}\text { Muito Importante } \\
(4-5)\end{array}$ \\
\hline Economia & $32(15,7 \%)$ & $39(19,1 \%)$ & $133(65,2 \%)$ \\
\hline $\begin{array}{l}\text { Comodidade e } \\
\text { centralização da inf. }\end{array}$ & $18(8,8 \%)$ & $43(21,1 \%)$ & $143(70,1 \%)$ \\
\hline Fiabilidade & $17(8,3 \%)$ & $44(21,6 \%)$ & $143(70,1 \%)$ \\
\hline Segurança & $0(0 \%)$ & $57(27,9 \%)$ & $147(72,1 \%)$ \\
\hline Flexibilidade & $28(13,7 \%)$ & $39(19,1 \%)$ & $137(67,2 \%)$ \\
\hline Abstração & $79(38,7 \%)$ & $36(17,7 \%)$ & $89(43,6 \%)$ \\
\hline $\begin{array}{c}\text { Colaboração e } \\
\text { controlo de versões }\end{array}$ & $96(47,1 \%)$ & $10(4,9 \%)$ & $98(48 \%)$ \\
\hline $\begin{array}{l}\text { Capacidade de } \\
\text { armazenamento }\end{array}$ & $13(6,4 \%)$ & $30(14,7 \%)$ & $161(78,9 \%)$ \\
\hline $\begin{array}{l}\text { Atualizações } \\
\text { automáticas }\end{array}$ & $7(3,4 \%)$ & $12(5,9 \%)$ & $185(90,7 \%)$ \\
\hline
\end{tabular}

De salientar o facto de a segurança figurar tanto nas vantagens mais importantes como nas desvantagens. Esta preocupação recai na segurança da adoção destes serviços e nas suas vulnerabilidades e perda de dados ou informações. Contudo, é importante referir que, quando falamos em vulnerabilidades de segurança, não nos referimos a falhas ou problemas nos serviços que permitam comprometer os dados e informações das organizações, mas sim à confiança que as pessoas têm nesses serviços, uma vez que há sempre um maior receio de perda quando os dados são guardados por terceiros.

Maioritariamente, as PME's não possuem uma área de TI tão ampla e poderosa capaz de assegurar completamente a integridade dos dados, assim, é fundamental a utilização de serviços de cloud computing, onde os níveis de segurança são maiores, para garantir maior qualidade no armazenamento dos dados e na tomada de decisões estratégicas relacionadas com os mesmos (Dias et al., 2012). 
Tabela 8. Importância das desvantagens do cloud computing

\begin{tabular}{|c|c|c|c|}
\hline Vantagens & $\begin{array}{c}\text { Pouco Importante } \\
\qquad(1-2)\end{array}$ & $\begin{array}{l}\text { Importante } \\
\text { (3) }\end{array}$ & $\begin{array}{l}\text { Muito Importante } \\
(4-5)\end{array}$ \\
\hline $\begin{array}{l}\text { Dependência de } \\
\text { internet }\end{array}$ & $56(27,5 \%)$ & $53(26 \%)$ & $95(46,5 \%)$ \\
\hline $\begin{array}{c}\text { Perda de } \\
\text { dados/informação }\end{array}$ & $22(11,3 \%)$ & $22(11,3 \%)$ & $150(77,4 \%)$ \\
\hline $\begin{array}{c}\text { Opções de } \\
\text { desenvolvimento de } \\
\text { software limitadas }\end{array}$ & $97(47,6 \%)$ & $68(33,3 \%)$ & $39(19,1 \%)$ \\
\hline $\begin{array}{c}\text { Vulnerabilidades de } \\
\text { Segurança }\end{array}$ & $37(18,1 \%)$ & $31(15,2 \%)$ & $136(66,7 \%)$ \\
\hline
\end{tabular}

\section{CONCLUSÃO}

Através do estudo realizado, podemos perceber que a virtualização e o cloud computing precisam de ser mais divulgados em Portugal, pois ainda existe um número muito elevado de empresas que não estão familiarizadas com os seus conceitos, vantagens e potencialidades. Ainda assim, praticamente todos os inquiridos que utilizam técnicas de virtualização e serviços de cloud se mostraram satisfeitos com os serviços, custos, possibilidades que os mesmos lhe proporcionaram e com o desenvolvimento do seu negócio, o que é determinante para se poder obter uma conclusão positiva deste estudo.

É ainda de notar que a adoção a este tipo de tecnologias e serviços é relativamente recente, o que por um lado demonstra o começo de uma transformação mas por outro lado, as empresas não aderentes ou que não estão familiarizadas com os conceitos, vantagens e desvantagens da virtualização e do cloud computing mostraram-se bastante reticentes, maioritariamente devido aos custos e ao receio de perda de informação importante, o que revela alguma dúvida em enveredar por novos caminhos por parte das organizações, quer das mais recentes com menos poder financeiro, quer das mais antigas, mais receosas face à mudança.

Concluindo, conseguiu-se despertar algum interesse nas PME's através dos inquéritos e da informação presente nos mesmos, o que é um bom indicador da disponibilidade das organizações a uma possível mudança de paradigma.

\section{REFERÊNCIAS}

Coelho, F. d. A., Calzavara, G. S., \& di Lucia, R. (2008). "Virtualização - VMWare e Xen." https://www.gta.ufrj.br/grad/09_1/versao-final/virtualizacao/origem\%20da\%20necessidade.html (acedido em January 26, 2019).

Dias, J. M. F., Rodrigues, R. d. C. M. C., \& Pires, D. F. (2012). A Segurança de Dados na Computação em Nuvens nas Pequenas e Médias Empresas. Revista Eletrónica de Sistemas de Informação e Gestão Tecnológica, 2, 56-69.

Fortes, N., Pereira, J. H., \& da Costa, J. F. (2016). A adoção de serviços cloud computing pelas empresas portuguesas: O papel dos esforços de marketing. RISTI - Revista Ibérica de Sistemas e Tecnologias de Informação(18). doi:10.17013/risti.18.33-48

Giraldo, V. (2018). "Cloud Computing: o que é, para que serve e como usar no seu negócio?" https://marketingdeconteudo.com/cloud-computing/ (acedido em November 14, 2018).

Gong, C., Liu, J., Zhang, Q., Chen, H., \& Gong, Z. (2010). The Characteristics of Cloud Computing. Paper presented at the 39th International Conference on Parallel Processing Workshops, San Diego.

Lavrador, M., \& Gouveia, A. J. (2020). Virtualization and Cloud Computing - Concepts. Paper presented at the 18th International Conference on e-Society, Sofia, Bulgaria.

Ruschel, H., Zanotto, M. S., \& da Mota, W. C. (2010). Computação em Nuvem. 15.

Saleem, M. (2017). Cloud Computing Virtualization. International Journal of Computer Applications Technology and Research, 6(7), 290-292. 